
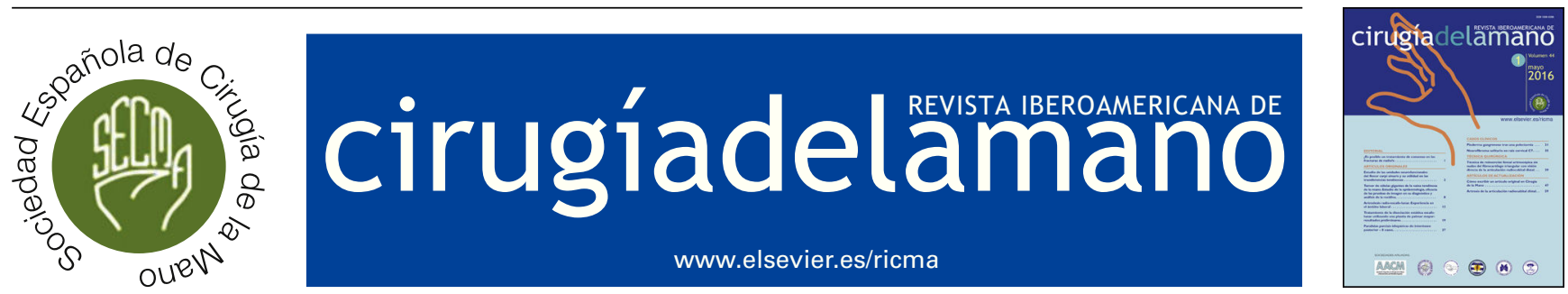

ARTÍCULO ORIGINAL

\title{
Estudio anatómico de la vascularización peroneal y su importancia clínica para el injerto vascularizado de peroné
}

\author{
M.R. Morro Martía,b,* , D. Gutiérrez Medina ${ }^{a}$, G.J. Tarnawski Español ${ }^{a}$, M. Llusá Pérez ${ }^{a, b}$ \\ y J.R. Ballesteros Betancourt ${ }^{a, c}$
}

a Laboratorio de Macro-Microdisección y Anatomía Quirúrgica, Departamento de Anatomía y Embriología Humana, Facultad
de Medicina, Universidad de Barcelona, Barcelona, España
b Servicio de Cirugía Ortopédica y Traumatología, Hospital de Traumatología Vall d’Hebron, Barcelona, España
' Servicio de Cirugía Ortopédica y Traumatología, Hospital Clínic de Barcelona, Barcelona, España

Recibido el 7 de enero de 2016; aceptado el 23 de julio de 2016

Disponible en Internet el 15 de septiembre de 2016

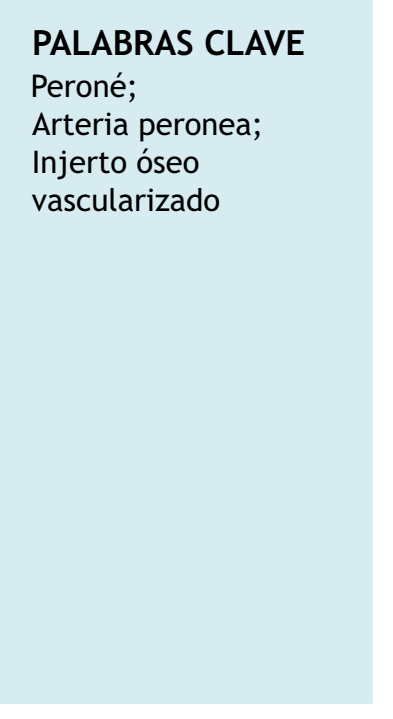

\begin{abstract}
Resumen
Introducción: Desde que Ueba utilizara por primera vez el injerto óseo vascularizado de peroné en 1973, esta técnica se ha usado con éxito como método de reconstrucción de múltiples tipos de defectos óseos. La correcta vascularización del injerto es clave para su supervivencia. El objetivo de este trabajo es describir la vascularización diafisaria y epifisaria proximal del peroné.

Material y método: Se utilizaron 12 especímenes de cadáver criopreservado inyectados con látex negro. Se procedió a su disección, seguida de una técnica de corrosión en solución alcalina para demostrar la distribución de la red vascular del peroné de forma precisa.

Resultados: Observamos que el peroné recibe su irrigación sobre todo de una abundante red de vasos periósticos en la diáfisis y en menor medida de la arteria nutricia. Apreciamos que la epífisis proximal presenta entre 2 y 6 ramos recurrentes, procedentes de las arterias tibial anterior y tronco tibioperoneo.

Conclusión: El aporte vascular perióstico de la diáfisis del peroné es el mayoritario, siendo limitada la vascularización a través de la arteria nutricia. En la epífisis proximal, la presencia de vasos recurrentes accesorios puede ser de relevancia durante la cirugía.

(C) 2016 SECMA. Publicado por Elsevier España, S.L.U. Este es un artículo Open Access bajo la licencia CC BY-NC-ND (http: / / creativecommons.org/licenses/by-nc-nd/4.0/).
\end{abstract}

\footnotetext{
* Autora para correspondencia.

Correo electrónico: mrmorro@ub.edu (M.R. Morro Martí).
} 


\section{KEYWORDS}

Fibula;

Fibular artery; Vascularised Bone Graft
Anatomical study of the peroneal vascularization and its clinical significance for vascularized fibular graft

\begin{abstract}
Introduction: Since Ueba performed the first vascularised fibular graft in 1973, this technique has become a useful method for the reconstruction of many bone defects. A proper graft vascularization is essential for its survival. The objective of this work is to describe the vascular anatomy of the diaphysis and proximal epiphysis of the fibula.

Material and methods: The arterial network of 12 cryopreserved cadaver specimens were injected with black latex. After their dissection, the specimens were immersed in a basemedium solution to process them with a corrosion technique, obtaining a precise distribution of the vascular network of the fibula.

Results: It was observed that the fibular diaphysis is mainly nourished by a rich network of periosteal vessels and partially by a nutrient artery. Between 2 and 6 recurrent vessels from the anterior tibial artery and the tibial-fibular trunk were observed nourishing the proximal epiphysis of the fibula.

Conclusion: The periosteal network supplying the fibula is the most important, while the nutrient artery is a secondary blood supply to the fibula. In the proximal epiphysis, there are accessory recurrent vessels which should be taken into account during surgery.

(c) 2016 SECMA. Published by Elsevier España, S.L.U. This is an open access article under the CC BY-NC-ND license (http://creativecommons.org/licenses/by-nc-nd/4.0/).
\end{abstract}

\section{Introducción}

La reconstrucción de defectos óseos grandes ha sido siempre un desafío para los cirujanos. Algunas de las técnicas más utilizadas para reconstruir estos defectos son los injertos ${ }^{1}$, tanto los aloinjertos como los autoinjertos. De estos últimos destacan los injertos vascularizados de peroné, tanto por la baja morbilidad de la zona donante como por su versatilidad en la colocación del hueso; además de poder aportar a la vez cobertura cutánea o muscular en los defectos complejos. La técnica fue inicialmente descrita por Ueba en $1973^{2}$, pero fue popularizada por Taylor en $1975^{3}$. Desde entonces, esta técnica ha sido modificada por Gilbert en $1979^{4}$ y ha inspirado otras como la de Innocenti en $2005^{5}$, que utiliza la epífisis proximal en edades pediátricas cuando se necesita una fisis abierta.

El éxito de los injertos óseos vascularizados radica en que las células óseas pueden sobrevivir a la transferencia y, junto a otros factores, favorecen teóricamente la consolidación. En el injerto no vascularizado, en cambio, el hueso funciona básicamente como un osteoconductor. Esta diferencia puede no ser significativa en defectos óseos pequeños, pero cuanto más largo es el defecto (sobre todo a partir de los $6 \mathrm{~cm}$ ), más riesgo hay de no incorporación del injerto si este no está vascularizado ${ }^{6}$. Se ha descrito que los injertos vascularizados de peroné se pueden basar en la arteria peronea cuando se utiliza la diáfisis o en la arteria tibial anterior si es la epífisis proximal la que se toma.

Aunque la anatomía vascular de la región ha sido ampliamente estudiada ${ }^{7,8}$, creemos que todavía existe espacio para una descripción más orientada a su uso clínico. Por lo tanto, debido a la importancia de una correcta irrigación para la supervivencia del injerto vascularizado de peroné, creemos que un estudio anatómico descriptivo de su vascularización podría ser de interés. Consideraremos las
2 técnicas más utilizadas, que son el injerto vascularizado de diáfisis peroneal basado en la arteria peronea descrita por Gilbert ${ }^{4}$ y el injerto vascularizado de epífisis proximal basado en la arteria tibial anterior descrito por Innocenti ${ }^{5}$.

El objetivo de este trabajo es describir la vascularización arterial de la epífisis proximal y la diáfisis del peroné y comentar las posibles implicaciones quirúrgicas en los injertos vascularizados de peroné.

\section{Material y método}

Se utilizaron 12 especímenes de cadáver humano criopreservado, con el árbol arterial inyectado con látex negro a través de la arteria femoral. Se tuvo en cuenta que ninguno de los especímenes mostrara signos de fracturas previas $u$ otras enfermedades que hubieran podido modificar la vascularización del hueso.

Se procedió a su disección anatómica. En primer lugar, se realizó una incisión entre los músculos tibial anterior y extensor largo de los dedos por donde se accedía a la arteria tibial anterior. Seguidamente, se desarrollaba el intersticio entre el compartimento lateral y el compartimento posterior de la pierna para visualizar la arteria peronea. En ese momento se seccionaba la membrana interósea, con cuidado de no dañar las arterias; y se hacía una osteotomía distal a nivel metafisario del peroné. Se hacía una artrotomía de la articulación tibioperonea proximal y se liberaban las partes blandas distales de la zona de la osteotomía y proximalmente a nivel del vértice de la cabeza del peroné. Finalmente se rechazaba el tejido muscular hasta dejar aproximadamente menos de $0,5 \mathrm{~cm}$ cubriendo el peroné. Se obtenía así una pieza anatómica de epífisis proximal y diáfisis peroneal con una pequeña cobertura muscular y 


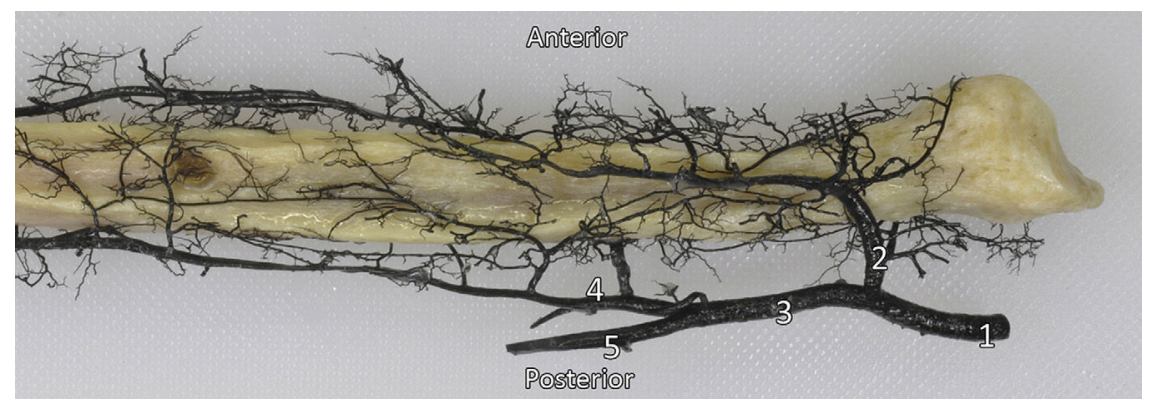

Figura 1 Preparación anatómica de la distribución de los vasos arteriales del peroné. 1: Arteria poplítea; 2: Arteria tibial anterior; 3: Tronco tibioperoneo; 4: Arteria peronea; 5: Arteria tibial posterior (seccionada).

un pedículo vascular correspondiente a las arterias tibial anterior, peronea y parte de la tibial posterior (fig. 1).

Seguidamente se procesaron las piezas con la técnica de corrosión en medio alcalino descrita por Tompsett ${ }^{9}$. Se sumergían los especímenes en una solución de hipoclorito de sodio, que se cambiaba cada 2 días, limpiando además el recipiente de grasa y otros restos orgánicos para que la base no reaccionase con estos en lugar de con el espécimen. El proceso duró aproximadamente una semana para cada pieza, aunque cada espécimen requirió una duración ligeramente diferente ( \pm 1 día) en función del volumen de tejido blando preservado en el momento de la disección. El resultado final fue la conservación exclusivamente del látex y el tejido óseo, que debían manipularse con cuidado por la facilidad con la que se pueden desprender los vasos del soporte óseo.

Finalmente, se midieron las distancias desde el orificio nutricio al vértice de la cabeza del peroné y al origen de la arteria peronea. También se midió el diámetro de la arteria nutricia. Además, se cuantificó el número de vasos periósticos de la diáfisis, y el número de anastomosis de más de $1 \mathrm{~mm}$ entre las arterias peronea y tibial anterior. Finalmente, se cuantificó el número de vasos recurrentes de la epífisis proximal y, centrándonos en la arteria tibial anterior, se midió la distancia desde la división de esta arteria hasta la emisión de las ramas recurrentes. Todas las medidas se realizaron con un pie de rey Series 500 Digimatic Absolute Caliper (Mitutoyo UK Ltd., Andover, Hampshire, Reino Unido). Al final del proceso de corrosión se realizó una serie fotográfica de cada pieza.

Se decidió usar la mediana y el rango intercuartílico como medida de dispersión debido a la pequeña muestra del estudio.

\section{Resultados}

Se observó que la diáfisis peroneal recibe su irrigación a través de la arteria peronea en forma de una arteria nutricia de pequeño calibre. Encontramos un peroné con 2 arterias nutricias $(8,3 \%)$ y otro sin ninguna $(8,3 \%)$. En todos los casos el diámetro de la arteria nutricia fue menor de $1 \mathrm{~mm}$. El foramen nutricio se localizó en todos los casos en la cara posterior del tercio medio del peroné, a una mediana de $14,7 \mathrm{~cm}(14,1 ; 17,4)$ del vértice de la cabeza del peroné (fig. 2). Además, encontramos que la arteria perforaba la cortical con una dirección distal en todos los especímenes (fig. 3).

Por otro lado, se observó una mediana de 5 vasos $(4 ; 6)$ procedentes de la arteria peronea, que con una distribución segmentaria formaban una rica red perióstica en la zona diafisaria (fig. 4). La arteria tibial anterior contribuía a la vascularización perióstica con una mediana de 2 anastomosis $(1 ; 2)$ de calibre superior a $1 \mathrm{~mm}$ entre esta y la arteria peronea.

Se observó una mediana de 3 vasos recurrentes $(2 ; 3)$ procedentes de la arteria tibial anterior para la epífisis proximal, aunque en total describimos 5 tipos de vasos recurrentes posibles. De proximal a distal, se desprendían un vaso recurrente posterior que se correspondería con la

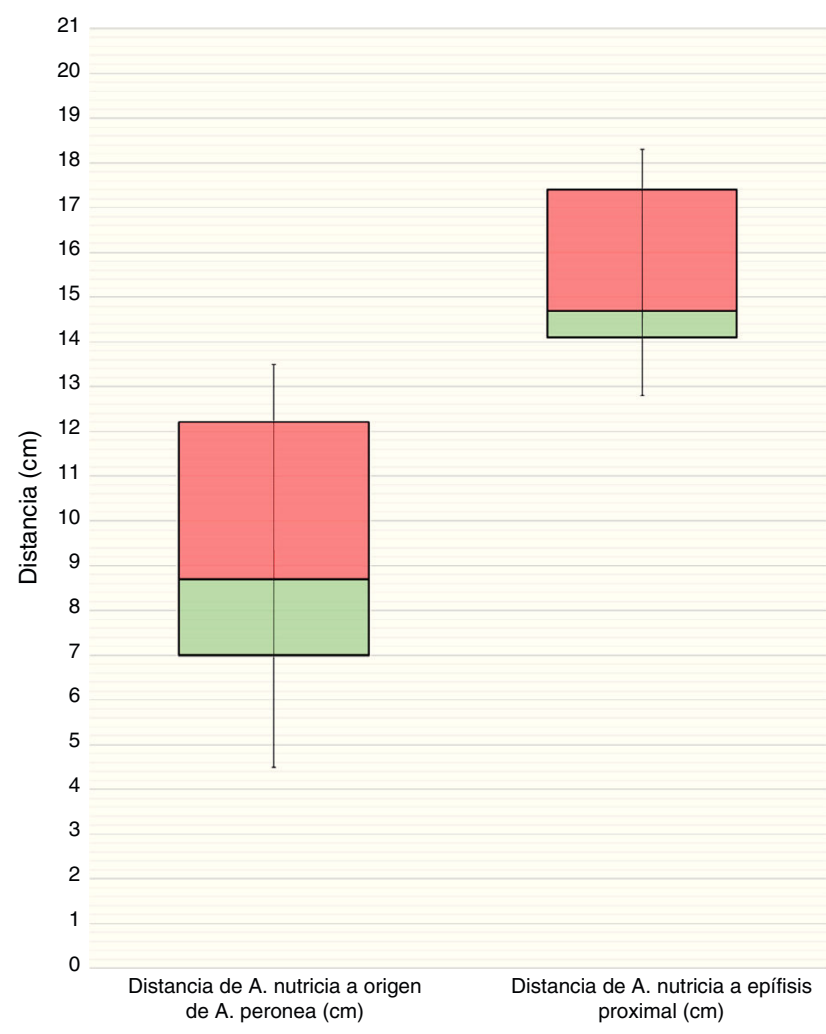

Figura 2 Diagrama de cajas de la distancia medida en centímetros de la arteria nutricia a vértice proximal y origen de la arteria peronea. A: Arteria. 


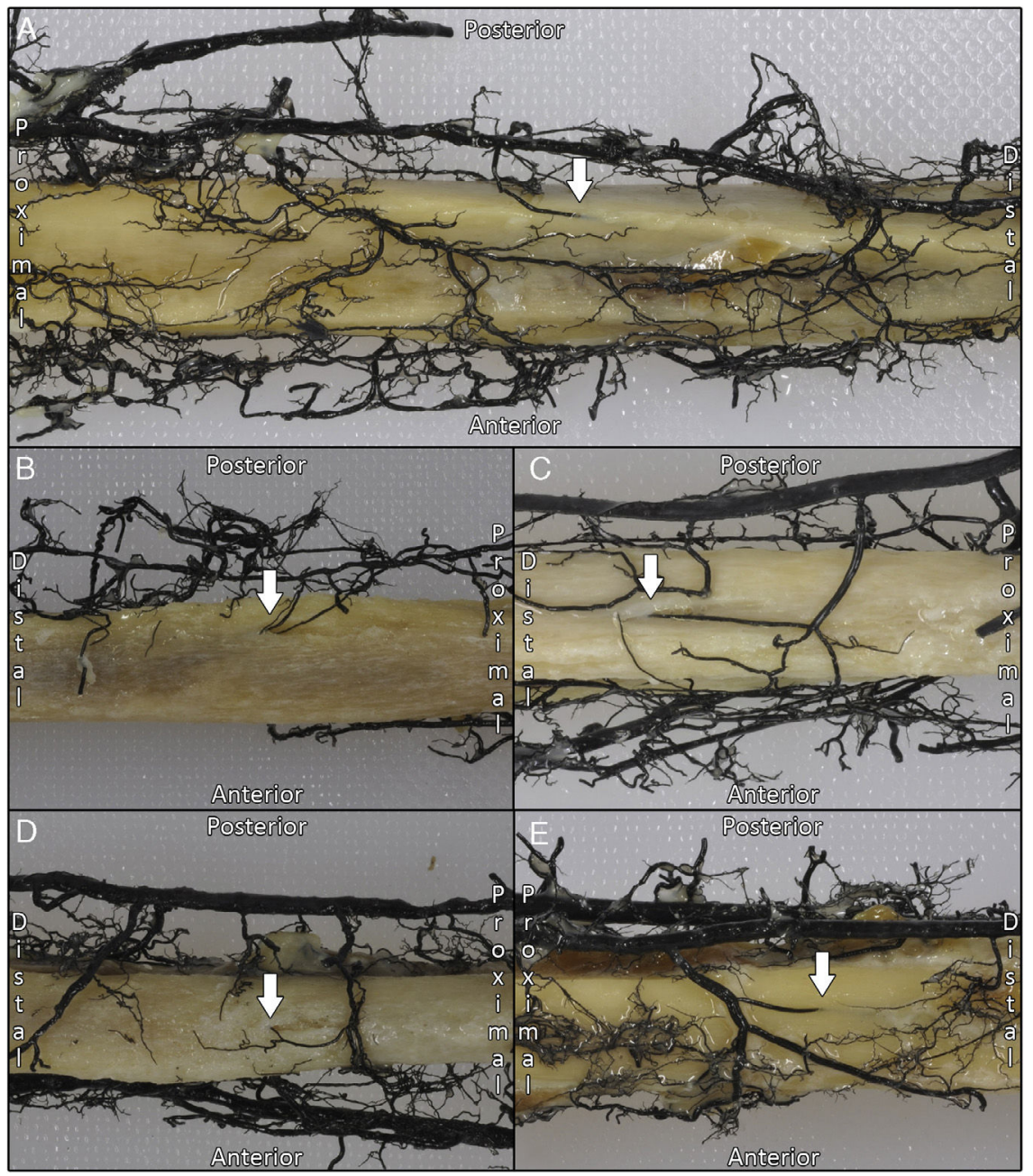

Figura 3 Preparaciones anatómicas de las arterias nutrícias de 5 peronés, indicadas con una flecha. Puede observarse su pequeño calibre relativo a los vasos periósticos que las rodean.

arteria recurrente tibial posterior, 2 ramos longitudinales paralelos al eje del peroné que se originaban cuando la arteria tibial anterior cruzaba el borde proximal de la membrana interósea, y 2 vasos recurrentes con dirección oblicua de distal a proximal y de medial a lateral que se corresponderían con una arteria recurrente tibial anterior y una arteria recurrente tibial anterior accesoria (figs. 5 y 6).

Dos de estas arterias recurrentes fueron visualizadas en todos los especímenes: una rama longitudinal que se encontró a $1 \mathrm{~cm}$ de mediana $(0,9 ; 1,2)$ de la división de la arteria tibial anterior y otra a $1,8 \mathrm{~cm}$ de mediana $(1,5 ; 2,2)$, que se correspondería con la arteria recurrente tibial anterior clásicamente descrita en la literatura.

En referencia a la vascularización posterior de la epífisis proximal del peroné, se observó que la arteria recurrente tibial posterior proviene de la arteria tibial anterior en 2 casos $(16,6 \%)$ y en 8 casos $(75 \%)$ proviene del tronco tibioperoneo. En 2 casos (16.6\%) no se observó arteria recurrente tibial posterior. Además, existía en 2 especímenes $(16.6 \%)$ una arteria recurrente tibial posterior accesoria procedente del tronco tibioperoneo o de la arteria poplítea que irrigaba la superficie posterior de la epífisis proximal (fig. 6).

\section{Discusión}

Según los resultados de nuestro estudio, el peroné se nutre básicamente de los abundantes vasos periósticos con un patrón segmentario, procedentes sobre todo de la arteria peronea y en menor medida de la arteria tibial anterior, formando una rica red vascular. Por el contrario, encontramos que la arteria nutricia del peroné es de un calibre muy pequeño, en todos los casos inferior a $1 \mathrm{~mm}$, como ya había comentado Zhong-wei ${ }^{1}$. Algunos estudios, no obstante, describen arterias nutricias de un tamaño superior, como Kokabiyik $^{10}$, que observó un diámetro medio de estas arterias de 1,2 $\mathrm{mm}$.

Observamos, además, variaciones en el número de arterias nutricias, concordantes con lo ya descrito en la literatura. Concretamente, en nuestro estudio encontramos un $8,3 \%$ de peronés con 2 arterias nutricias, resultados parecidos a los de Kizilkanata et al. ${ }^{11}(6,6 \%)$ y Sendemir y Cimen ${ }^{12}$ $(7,2 \%)$. En cambio, Prashanth et al. ${ }^{13}(0 \%)$, Pereira et al. ${ }^{14}$ $(0,87 \%)$ Guo $^{15}(3,3 \%)$ y Kokabiyik ${ }^{10}(5,4 \%)$ encontraron porcentajes inferiores; y Gümüsburun et al. ${ }^{16}(11,6 \%)$ y Menck y Sander ${ }^{17}(15,5 \%)$ obtuvieron porcentajes superiores a los encontrados por nosotros. 


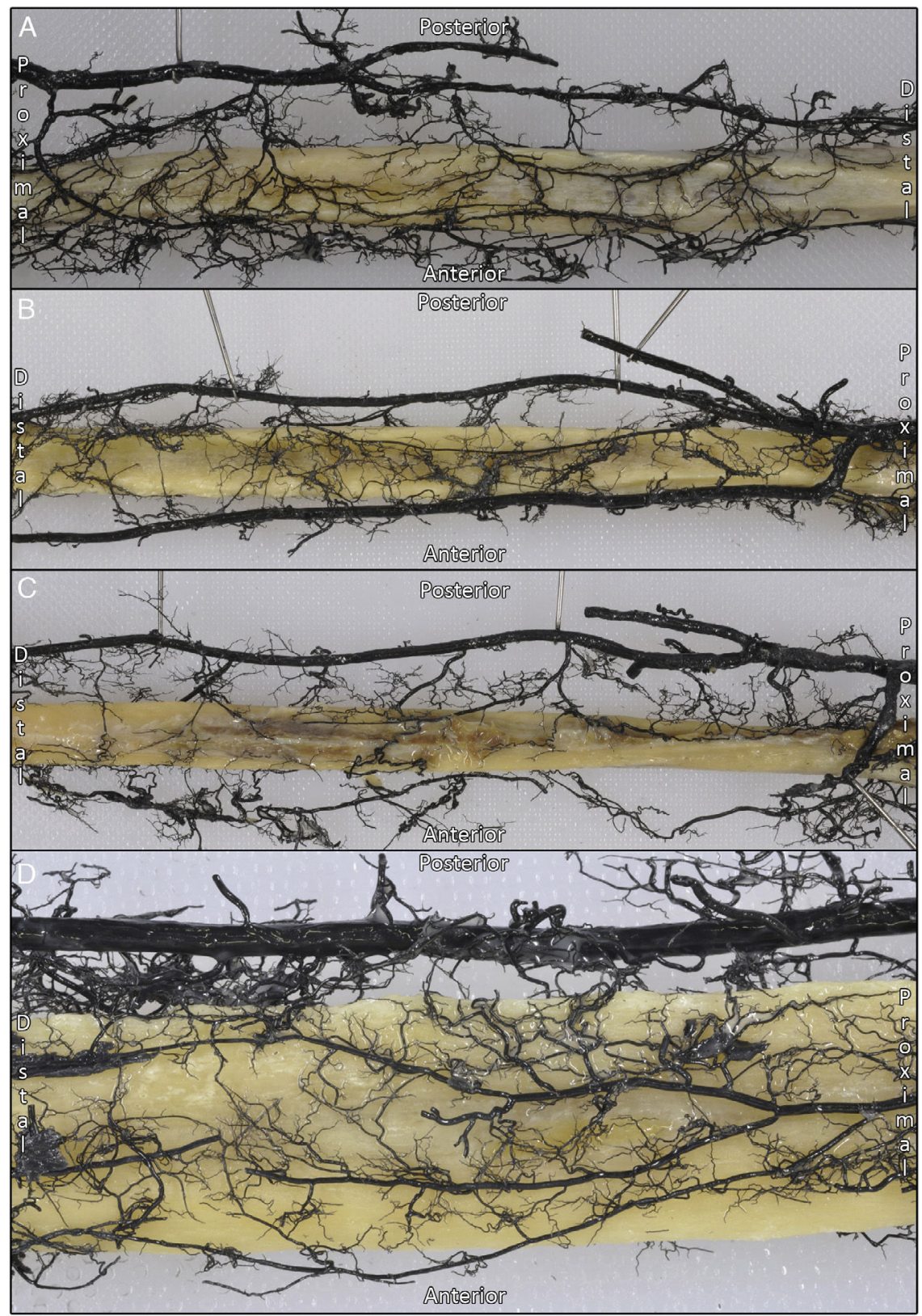

Figura 4 Preparaciones anatómicas de la distribución de la circulación perióstica en 4 especímenes.

Además observamos un $8,3 \%$ de peronés sin arteria nutricia, que coincide con lo observado por Prashanth et al. ${ }^{13}$ $(9,8 \%)$. Pereira y Lopes ${ }^{14}(0 \%)$, Menck y Sander ${ }^{17}(1 \%)$, Kizilkanata et al. ${ }^{11}(1,3 \%)$, Guo $^{15}(1,7 \%)$, Kokabiyik ${ }^{10}(3,3 \%)$ y Gümüsburun et al. ${ }^{16}(3,3 \%)$, que encontraron porcentajes inferiores a los nuestros, mientras que los de Sendemir y Cimen $^{12}(18,9 \%)$ fueron superiores.

En cuanto a la localización del foramen nutricio, los resultados de este estudio se correlacionan más con los de Kizilkanata et al. ${ }^{11}$, Gümüsburun et al. ${ }^{16}$ y Forriol Campos et al. ${ }^{18}$, que encontraron también la cara posterior como la posición predominante del foramen nutricio. Por el contrario, se oponen a los resultados de Kokabiyik et al. ${ }^{10}$, Prashanth et al. $^{13}$ y Sendemir y Cimen ${ }^{12}$, que observaron con mayor frecuencia el foramen en la cara medial; y a los resultados de Pereira et al. ${ }^{14}$, que observaron con mayor frecuencia el foramen en la cara lateral. Respecto a su localización en el eje longitudinal del peroné, todos los autores excepto $\mathrm{Guo}^{15}$ localizan el foramen nutricio en el tercio medio, coincidiendo con nuestros resultados. En concreto, Kokabiyik et al. ${ }^{10}$ lo sitúan a una distancia media de $17,1 \mathrm{~cm}$ $(9,4 ; 18,8)$ del vértice de la cabeza del peroné.

Respecto a la red perióstica vascular del peroné, nuestros resultados coinciden con los descritos por Strauch y Yull $^{7}$ y Zhong-wei et al. ${ }^{1}$, que describen la presencia de 4-6 vasos periósticos. Además, Menck y Sander ${ }^{17}$ ya comentan la importancia de esta red, y de cómo esta es dependiente de la arteria tibial anterior en el tercio proximal del peroné y de 
5

4

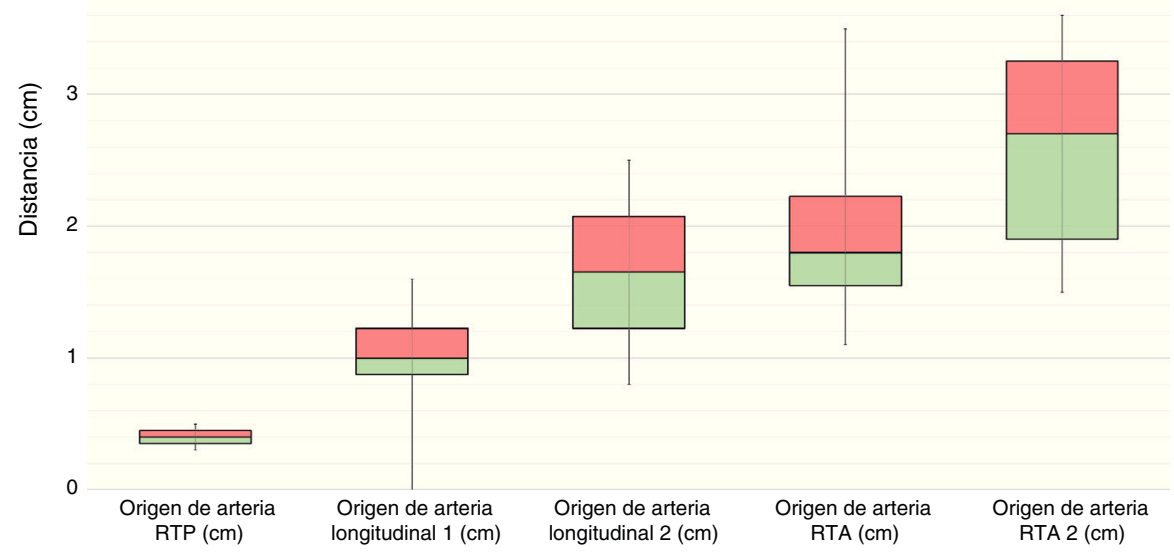

Figura 5 Diagrama de cajas de la distancia de la división de la arteria tibial anterior a la emisión de los ramos recurrentes medida en centímetros. RTA: recurrente tibial anterior; RTP: recurrente tibial posterior.

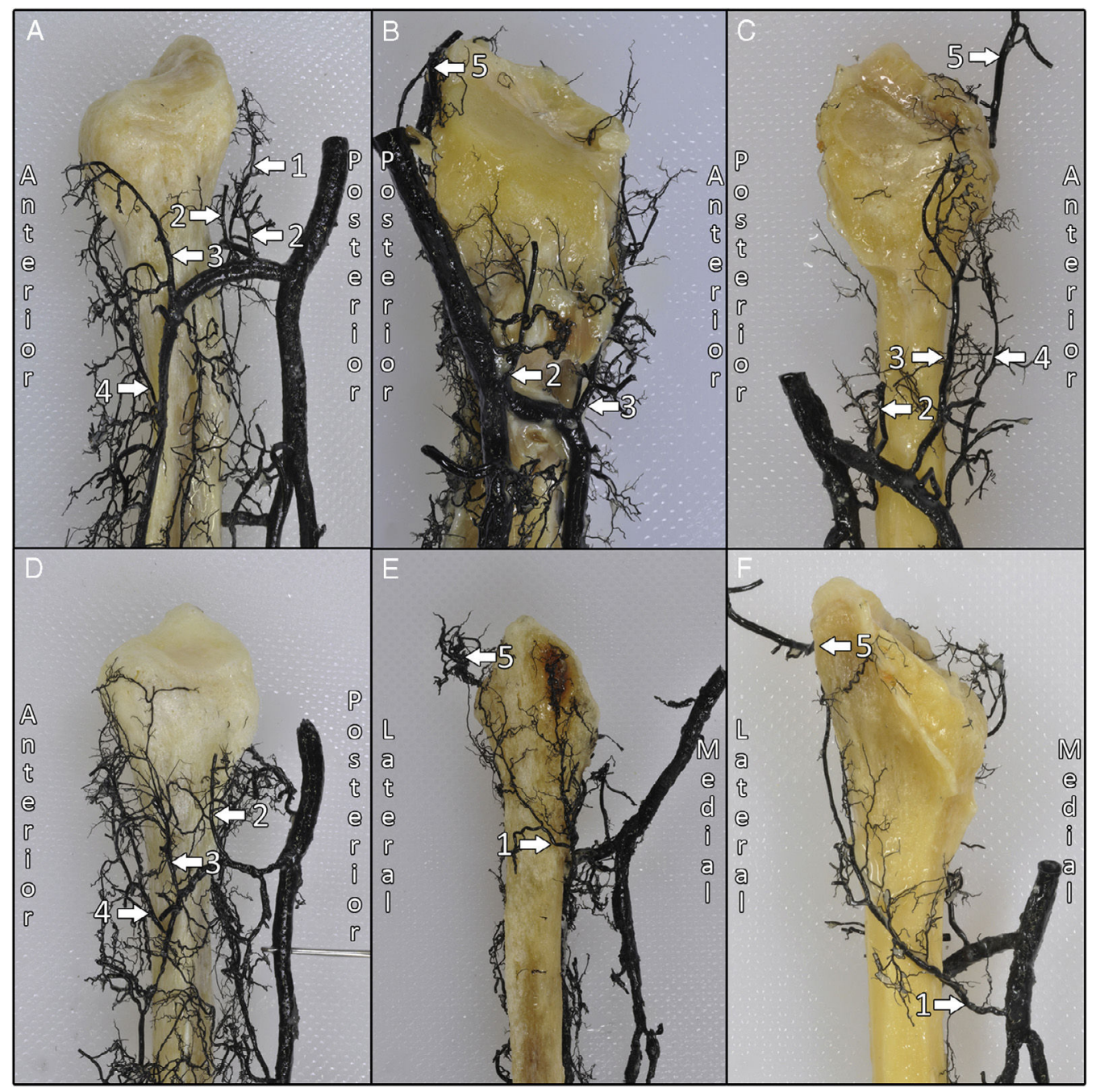

Figura 6 Preparaciones anatómicas de la distribución de los vasos que nutren la epífisis proximal de 6 peronés. 1: Arteria recurrente tibial posterior; 2: arterias longitudinales; 3: arteria recurrente tibial anterior; 4: arteria recurrente tibial anterior; 5: arteria inferolateral de la rodilla. 
la arteria peronea en los 2 tercios distales. También describen anastomosis importantes entre la arteria tibial anterior y peronea concordantes con las encontradas en nuestro estudio.

A nivel de la epífisis proximal observamos que la arteria recurrente tibial posterior puede desprenderse de la arteria tibial anterior o del tronco tibioperoneo. Concretamente encontramos que el $16,6 \%$ de estas procedían de la arteria tibial anterior y el $75 \%$ del tronco tibioperoneo. Estos resultados coinciden con los de Thammaroj et al. ${ }^{19}$ (33 y $72,7 \%$ respectivamente) pero se oponen a los resultados de Mozaffarian et al. ${ }^{20}$ (75 y $25 \%$ respectivamente).

No hemos encontrado bibliografía que describa vasos a nivel de la membrana interósea (los referidos como «longitudinal» en nuestro estudio) ni la presencia de arterias recurrentes tibial anterior y tibial posterior accesorias. Tampoco hemos encontrado ningún trabajo donde midan la distancia desde la división de la arteria tibial anterior hasta la emisión de cada uno de los vasos recurrentes.

Las principales limitaciones de este estudio son: el pequeño tamaño de la muestra y el sesgo poblacional, ya que todas las piezas anatómicas pertenecían a personas de edad avanzada, con una media de 78,6 años; y se sabe que la edad puede afectar a la vascularización ósea.

\section{Conclusión}

Según nuestros resultados, la arteria nutricia parecería ofrecer un aporte vascular menor al peroné, y su ausencia posiblemente no afectaría a la viabilidad de este. Esta idea se ve reforzada también por la abundante presencia de vasos periósticos, por el calibre de estos (iguales o superiores a los de la arteria nutricia) y por la presencia de variaciones anatómicas sin arteria nutricia.

Por lo tanto, nos parece importante mantener una mínima cobertura muscular del hueso a la hora de levantar un injerto vascularizado de peroné que asegure la preservación de esta red perióstica. De todas maneras, si la zona receptora no permite este pequeño volumen añadido, no es imprescindible para la viabilidad del injerto vascularizado, si se tiene cuidado con preservar la red vascular perióstica. También creemos que, según nuestros resultados, pueda no ser necesario localizar la arteria nutricia durante la cirugía, ya que podría dañar la red vascular perióstica.

En cuanto a la epífisis proximal, la presencia o no de los vasos recurrentes accesorios descrita no supone ninguna modificación de la técnica, ya que habitualmente se deja un pequeño remanente muscular para proteger los vasos recurrentes de la arteria tibial anterior. No obstante, resulta interesante conocer la presencia de la arteria recurrente tibial posterior y posibles ramos accesorios, ya que durante el levantamiento del injerto vascularizado de epífisis proximal podrían sangrar.

Según nuestros resultados, por la considerable variabilidad encontrada en los vasos recurrentes, podría ser de utilidad realizar una angiografía preoperatoria en estos pacientes, para conocer de antemano las variaciones individuales, que deberían ser tenidas en cuenta durante la cirugía.

\section{Responsabilidades éticas}

Protección de personas y animales. Los autores declaran que los procedimientos seguidos se conformaron a las normas éticas del comité de experimentación humana responsable y de acuerdo con la Asociación Médica Mundial y la Declaración de Helsinki.

Confidencialidad de los datos. Los autores declaran que en este artículo no aparecen datos de pacientes.

Derecho a la privacidad y consentimiento informado. Los autores han obtenido el consentimiento informado de los pacientes y/o sujetos referidos en el artículo. Este documento obra en poder del autor de correspondencia.

\section{Conflicto de intereses}

Los autores declaran no tener ningún conflicto de intereses.

\section{Bibliografía}

1. Zhong-wei C, Dong-yue Y, Di-sheng C. Microsurgery. New York: Springer; 1982.

2. Ueba Y, Fujikawa S. Nine years follow-up of a vascularized fibular graft in neurofibromatosis. A case report and literatura review. Orthop Surg Traumatol (Jpn). 1983;26:595-600.

3. Taylor GI, Graeme D, Miller H. The free vascularized bone graft: A clinical extension of microvascular techniques. Plast Reconst Surg. 1975;55:533.

4. Gilbert A. Vascularized transfer of the fibular shaft. Int J Microsurg. 1979;1:100-2.

5. Innocenti M, Delcroix L, Romano GF. Epiphyseal transplant harvesting technique of the proximal fibula based on the anterior tibial artery. Microsurgery. 2005;25:284-92.

6. Weiland AJ, Phillips TW, Randolph MA. Bone grafts: A radiologic, histologic, and biomechanical model comparing autografts, allografts, and free vascularized bone grafts. Plast Reconstr Surg. 1984; 74:368-79.

7. Strauch B, Yu HL. Atlas of microvascular surgery. New York: Theme; 1993.

8. Zenn MR, Jones G. Reconstructive surgery. Missouri: Quality Medical Publishing; 2012.

9. Tompsett DH. Anatomical techniques. Edinburgh and London: E\&S Livingstone; 1970.

10. Kokabiyik N, Yalçin B, Ozan H. Variations of the nutrient artery of the fibula. Clin Anat. 2007;20:440-3.

11. Kizilkanata E, Boyana N, Ozsahina ET. Location, number and clinical significance of nutrient foramina in human long bones. Ann Anat. 2007;189:87-95.

12. Sendemir E, Cimen A. Nutrient foramina in the shafts of lower limb long bones: Situation and number. Surg Radiol Anat. 1991;13:105-8.

13. Prashanth KU, Murlimanju BV, Prabhu LV. Morphological and topographical anatomy of nutrient foramina in the lower limb long bones and its clinical importance. Australas Med J. 2011;10:530-7.

14. Pereira GAM, Lopes PTC, Santos AMPV. Nutrient foramina in the upper and lower limb long bones: Morphometric study in bones of Southern Brazilian adults. Int J Morphol. 2011;29:514-20.

15. Guo F. Fibular blood supply. Chin Med J. 1981;94:396-404.

16. Gümüsburun E, Yucel $F$, Ozkan Y. A study of the nutrient foramina of lower limb long bones. Surg Radiol Anat. 1994;16:409-12. 
17. Menck J, Sander A. Periostale und intraossäre Versorgungsstrukturen der fibula und ihre klinische Bedeutung. Act Anat. 1992;145:400-5.

18. Forriol Campos F, Gomez Pellico L, Gianonatti Alias M. A study of the nutrient foramina in human long bones. Surg Radiol Anat. 1987;9:251-5.
19. Thammaroj T, Jianmongkol S, Kamanarong K. Vascular anatomy of the proximal fibula from embalmed cadaveric dissection. J Med Assoc Thai. 2007;90:942-6.

20. Mozaffarian K, Lascombes P, Dautel G. Vascular basis of free transfer of proximal epiphysis and diaphysis of fibula: An anatomical study. Arch Orthop Trauma Surg. 2009;129:183-7. 